\title{
Heterogeneidade do Diabetes Melito Tipo 1
}

\begin{abstract}
RESUMO
O diabetes melito tipo 1 (DM1) caracteriza-se pela deficiência de insulina por causa da destruição das células-beta pancreáticas. O DM1 atualmente é classificado em dois subtipos: um auto-imune (DM1A) e outro não auto-imune (DM1B). O DM1A poligênico (isolado ou associado a outras doenças autoimunes) é a forma mais prevalente. O DM1A pode fazer parte de síndromes raras em virtude de alterações monogênicas [gene regulador da auto-imunidade (AIRE)] e mutações no gene FOX-p3. O DM1B corresponde de $4 \%$ a $7 \%$ do DM1 e pode incluir formas não clássicas, como o diabetes fulminante e o DATC. Jovens com DM1A e sinais de resistência à insulina associados têm sido denominados de diabetes duplo (DD), tipo 1 e tipo 2. Nessa revisão são discutidas as patofisiologias e as características clínicas das formas raras de DM1A, o DM1B, as formas atípicas de DM1 não auto-imune e as inter-relações entre a inflamação subclínica da obesidade e o processo auto-imune do DM1A no DD. Em resumo, apresentamos o conceito de heterogeneidade do DM1. (Arq Bras Endocrinol Metab 2008;52/2:205-218)
\end{abstract}

Descritores: Diabetes melito do tipo 1; Heterogeneidade

\begin{abstract}
Heterogeneity of Type 1 Diabetes Mellitus.

Type 1 diabetes (T1D) comprises all forms of autoimmune-mediated and idiopathic beta-cell destruction leading to absolute insulin deficiency. The etiological heterogeneity of T1D has been recognized for the last decades, but it has been divided into only two subtypes so far: autoimmune (T1D)A and nonautoimmune (T1D)B mediated. Polygenic T1DA (isolated or associated to other autoimmune diseases) is the most prevalent type of T1D. T1DA might be part of rare monogenic syndromes related to mutations in the autoimmune regulator gene (AIRE) and FOXp3. Non-autoimmune forms of T1D correspond to approximately 4 to $7 \%$ of newly diagnosed T1D and include T1DB, as well as other types of atypical diabetes, for example fulminant type 1 diabetes and adult ketosis-prone diabetes. A new expression of diabetes in young with insulin resistance and obesity, along with the presence of pancreatic autoimmunity markers, namely auto-antibodies to islet cell antigens, is called double diabetes (DD), T1DA plus type 2 diabetes. Evidence has been collected concerning the potential effect of obesity-linked cytokines in amplifying the autoimmune response in DD. Therefore all these issues are presented and discussed in this review as the concept of heterogeneity of Type 1 Diabetes. (Arq Bras Endocrinol Metab 2008;52/2:205-218)
\end{abstract}

Keywords: Type 1 diabetes mellitus; Heterogeneity perspectiva

\author{
Sergio Atala Dib
}

Disciplina de Endocrinologia e

Centro de Diabetes do

Departamento de Medicina da

Universidade Federal de São

Paulo/Escola Paulista de

Medicina (Unifesp/EPM), SP, Brasil.
Recebido em 28/01/2008 Aceito em 01/02/2008 


\section{INTRODUÇÃO}

$\mathrm{O}$ DIABETES MELITO TIPO l (DMl) inclui todas as formas de diabetes em que ocorre primariamente a destruição das células-beta pancreáticas produtoras de insulina.

Na maioria dos casos, esta destruição é auto-imune e constitui o subgrupo de diabetes denominado de tipo lA (DMlA). Este subgrupo é composto de uma forma poligênica clássica (o tipo mais freqüente de DMl) (1), que pode apresentar-se isolado ou associado a outras endocrinopatias auto-imunes - síndromes poliglandulares auto-imunes 2 e 3 (2), do diabetes auto-imune latente do adulto (LADA) (3) e dos dois presentes em síndromes relacionadas a alterações monogênicas: um que pertence a síndrome poliglandular auto-imune do tipo 1 (SPAI-1) (4) e o associado à poliendocrinopatia e à enteropatia ligada ao $\mathrm{X}$ (IPEX) (5).

O subgrupo denominado tipo 1 idiopático é caracterizado por baixos níveis plasmáticos de insulina e de peptídeo-C, semelhantes ao do DMlA, embora não apresente evidência de auto-imunidade anticélulas-beta pancreáticas. A maioria desses pacientes, descritos até o momento, é de origem africana ou asiática. Estes podem apresentar episódios de cetoacidose diabética (CAD), mas a patogênese da sua insulinopenia permanece desconhecida. Este subgrupo é composto de pelo menos três subtipos: o diabetes melito tipo $1 \mathrm{~B}$ (DMIB) (6), o diabetes do adulto com tendência à cetose (DATC) (7) e o diabetes fulminante (8).

Uma publicação recente $(8 \mathrm{a})$ refere que entre as crianças e jovens com DMlB pode-se encontrar alguns indivíduos com mutações no gene da insulina. Estas alterações poderiam ser pesquisadas em casos selecionados desse grupo de diabetes.

O aumento da prevalência da obesidade na infância e na adolescência (9) e o diagnóstico mais precoce do DMIA (10) têm levado ao aparecimento de jovens com características de diabetes tipo 2 juntamente com a presença de auto-imunidade antipancreática. Estes pacientes têm sido denominados por alguns autores de diabetes duplo (DD) (11).

O DMlA poligênico clássico e o LADA estão sendo abordados em outros artigos desta edição da revista.

Aqui discutiremos os tipos monogênicos do DMIA, o DMIB, o diabetes fulminante, o DATC e o DD.

\section{SÍNDROME POLIGLANDULAR AUTO-IMUNE TIPO 1}

A SPAI-1, também conhecida como autoimmune polyendocrinopathy candidiasis ectodermal dystrophy (Apeced), é uma poliendocrinopatia rara em razão de mutações no gene regulador da auto-imunidade (AIRE). A freqüência dessas mutações varia em populações diferentes. A análise do gene AIRE para as mutações comuns é útil na identificação de indivíduos com SPAI-1, entretanto, como mutações múltiplas têm sido descritas, a ausência das mutações mais comuns podem não excluir o diagnóstico da SPAI-1.

A doença é transmitida por um modo de herança autossômico recessivo. O gene AIRE está localizado no cromossomo 2lq22.3 (12) e é importante para começar a transcrição de antígenos periféricos (normalmente expressos em tecidos fora do sistema imunológico) no interior de células epiteliais da região medular do timo (13). A expressão desses antígenos é importante durante a maturação tímica das células $\mathrm{T}$ e na seleção negativa das células desse tipo, que reagem com auto-antígenos.

A SPAI-1 é definida pela presença de dois ou três dos seguintes componentes: candidíase mucocutânea, insuficiência adrenal e/ou hipoparatiroidismo. Os indivíduos afetados geralmente desenvolvem candidíase mucocutânea persistente ou recorrente desde os primeiros anos de vida. Esta síndrome tem $25 \%$ de risco de recorrência para parentes de primeiro grau de indivíduos afetados (estes podem ser considerados afetados com apenas um dos componentes citados) e apresenta alta prevalência na Sardinia, na Finlândia e em famílias de judeus iranianos.

A candidíase mucocutânea e os anticorpos antiinterferon (14) estão presentes em 100\% desses casos.

O DMl está presente em $23 \%$ desses pacientes, isto é, em porcentagem bem menor do que a de outros componentes da SPAI-1, como a candidíase mucocutânea $(100 \%)$, hipoparatiroidismo $(86 \%)$ e insuficiência adrenal $(79 \%)$. Os auto-anticorpos anti-GAD 65 e antiIA2 apresentam sensibilidade, especificidade e valor preditivo diferentes para o diabetes da SPAI-1, quando comparados ao DMlA clássico (15). Os auto-anticorpos anti-IA2 parecem ser os de melhor valor preditivo para o diabetes na SPAI-l com nenhum ou pequeno valor adicional do anti-GAD65 (16). Isso pode sugerir que mecanismos fisiopatológicos diferentes estejam operando na destruição das células-beta e também na existência de outro auto-antígeno da célula-beta, até o momento desconhecido, na SPAI-1. 
Além das doenças citadas anteriormente, outras características clínicas (com as respectivas prevalências entre parênteses) da SPAI-l são: a falência ovariana (72\%), a displasia ectodérmica ( $50 \%$ a $75 \%$ ), a anemia perniciosa (31\%), o hipogonadismo masculino $(26 \%)$, a obstipação intestinal (21\%), o hipotiroidismo (18\%), a diarréia $(18 \%)$, a hepatite (18\%) e a atrofia esplênica (15\%). Estes estigmas devem ser seguidos nesses pacientes.

\section{SÍNDROME DA IMUNODISREGULAÇÃO POLIENDOCRINOPATIA ENTEROPATIA LIGADA AO $\mathrm{X}$}

Esta síndrome, descrita pela primeira vez em 1982, é uma doença rara, recessiva, ligada ao $\mathrm{X}$ e que se caracteriza por disregulação imunológica que resulta doenças auto-imunes múltiplas e óbito prematuro.

O DMl precoce está entre as características clínicas dessa síndrome que inclui também uma enteropatia grave, a qual compromete o crescimento e o desenvolvimento. O eczema ou atopia, a trombocitopenia, a anemia hemolítica, o hipotiroidismo e a linfadenopatia também fazem parte dessa síndrome (17).

Estudos em modelos experimentais (18) da doença sugerem que células $\mathrm{T}$ reguladoras estejam envolvidas na sua patogênese.

O defeito genético que causa a IPEX foi identificado recentemente em famílias com a doença, e corresponde a mutações dentro do gene FOX-p3 (19). O gene FOX-p3 codifica a produção de uma proteína denominada scurfin e pertence a classe dos fatores de transcrição. O FOX-p3 está expresso nas células T reguladoras $\mathrm{CD}^{+} / \mathrm{CD}^{2} 5^{+}(20)$. Estas células $\mathrm{T}$ podem suprimir a ativação de outras células T. De modo que mutações no FOX-p3 podem resultar a incapacidade de gerar células T reguladoras e o desenvolvimento da IPEX com a auto-imunidade contra vários órgãos. $\mathrm{O}$ tratamento tem como objetivo as abordagens das diferentes patologias que compõem a síndrome. Como um todo, o transplante de medula óssea tem sido tentado com sucesso variável (21). O uso de terapêutica imunossupressora com sirolimo apresentou efeitos satisfatórios em três pacientes com IPEX (22).

\section{Diabetes melito tipo 1B}

Uma parcela de pacientes com DMl não apresenta evidência de auto-imunidade humoral contra as ilhotas pancreáticas ao diagnóstico clínico, pelo menos com os testes diagnósticos atuais mais comuns. Muitos destes pacientes podem apresentar forma clínica atípica da doença. Este subgrupo de pacientes com auto-anticorpos negativos são classificados como tipo $1 \mathrm{~B}$.

Aproximadamente $4 \%$ a $7 \%(23,24)$ dos pacientes com DMl recentemente diagnosticados são auto-anticorpos negativos.

Em um estudo americano recente (25), 859 crianças e jovens (idade de 0 a 18 anos) foram analisados, dentro das primeiras duas semanas de diagnóstico do diabetes, em relação aos auto-anticorpos antiilhotas, genótipos do HLA e HbAlc. Neste estudo aproximadamente $20 \%$ dos pacientes foram negativos para todos os quatro anticorpos pesquisados (antiinsulina, antiGAD, ICA512 (incluindo vários subtipos) e antiilhota clássico (ICA). A prevalência de indivíduos com ausência dos anticorpos antiilhota apresentou relação positiva com a idade, em todos os três grupos étnicos estudados (caucasianos, hispânicos e negros), sendo mais acentuada nestes dois últimos e com o índice de massa corpórea (IMC). A ocorrência dos auto-anticorpos (AAc) estava relacionada à presença do genótipo HLA de altíssimo risco (DR3-DQ2 e DR4-DQ8) para DMIA nessa população. Apenas $9 \%(9 / 105)$ das crianças portadoras deste genótipo HLA eram AAc- contra 20\% (66/336), entre outros genótipos HLA de classe II (p $=0,007)$. Por outro lado, $63 \%$ das crianças com alelo protetor $\left(\mathrm{DQBl}{ }^{*} 0602\right)$ eram AAc- $(\mathrm{p}<0,0001)$ contra apenas 1,6\% das crianças auto-AAc+.

A média do IMC entre as crianças com diabetes que eram anticorpos negativos foi significantemente maior do que o entre as crianças que eram anticorpos positivos $\left(23,3\right.$ versus $\left.18,9 \mathrm{~kg} / \mathrm{m}^{2} ; \mathrm{p}<0,001\right)$. Em relação aos valores de HbAlc, não houve diferença entre os pacientes com auto-anticorpos positivos e negativos ( 11,2 versus $11,3 \% ; \mathrm{p}=0,259)$.

Em resumo, este estudo conclui que uma porcentagem significante de maneiras não-auto-imunes de DMl pode estar presente em crianças de todos as faixas etárias com grande aumento após os 10 anos de idade, sugerindo heterogeneidade etiológica.

É importante ressaltar que nessas crianças e jovens com AAc - deve-se fazer o diagnóstico diferencial com o diabetes do tipo 2 do jovem, com maturity-onset diabetes of the young (MODY), mutações no DNA mitocondrial, síndrome de Wolfram, mutações do Kir6.2, como também de maneiras secundárias e endócrinas e algumas síndromes raras. 
Entretanto, esta área de estudo do DMlB está em constante evolução. Um estudo recente (26) mostrou que anticorpos contra um novo antígeno das ilhotas (ZnTA) foram encontrados em 26\% dos DMl classificados inicialmente AAc - com base nos marcadores existentes (GADA, IA2, IAA e ICA).

A combinação dos anticorpos anti-ZnT8A, GADA, IA2 e IAA é capaz de aumentar a detecção de autoimunidade para $8 \%$ no início do DMl.

\section{Diabetes fulminante}

Entre as formas de diabetes com deficiência primária de insulina não-mediada pela auto-imunidade faz parte $o$ denominado diabetes fulminante. Esta denominação foi proposta em 2000 por Imagawa e col. para um grupo de pacientes japoneses que apresentaram CAD associada a valores baixos de HbAlc (27). Casos semelhantes ao DMl fulminante já haviam sido descritos anteriormente por outro grupo japonês, em 1987 (28). O DMl fulminante apresenta características clínicas distintas do DMlA e é constituído por (29): 1. Desenvolvimento e evolução rápidos para $\mathrm{CAD} ; 2$. Tempo de duração dos sintomas de diabetes muito curto; 3 . Predominância do sexo masculino; 4. Início preponderante na vida adulta; 5. Precedência freqüente de sintomas gripais; 6. Dependência permanente da insulina exógena; 7 . Autoanticorpos antiilhotas pancreáticas negativos; 8 . Valores elevados de enzima pancreática sérica; 9. Infiltração linfocítica no pâncreas exócrino; e 10. Destruição das células-alfa e beta das ilhotas pancreáticas (30). Nas mulheres, a gestação também é fator de risco para o DMl fulminante.

Um estudo multicêntrico nacional sobre a coordenação da Sociedade Japonesa de Diabetes sugere que este subtipo de diabetes pode concorrer com aproximadamente $20 \%$ dos casos de DMl que se apresentam com cetose ou CAD (31) naquele país. Os critérios diagnósticos para o DMl fulminante propostos pelo
Comitê de Estudo do Diabetes Tipo l Fulminante no Japão estão apresentados na Tabela 1 .

A patogênese desse tipo de diabetes é praticamente desconhecida. Em relação aos fatores genéticos, os estudos mostram que apenas uma parte (DRBI * 1501 DQB $\left.{ }^{*} 0401\right)$ dos antígenos de classe II do sistema HLA (DRBI*1501-DQBI*0401 e DRBI*0901$\mathrm{DQB1}{ }^{*} 0303$ ), que são de risco para o $\mathrm{DMl}$ em caucasianos, contribuem para o desenvolvimento do DMl fulminante. Do mesmo modo, dois (DRB1*1502$\mathrm{DQB1}{ }^{*} 0601$ ou $\mathrm{DRB1}{ }^{*} 1501-\mathrm{DQB1}{ }^{* 0602)}$ dos maiores fatores do sistema HLA protetores para o DMl em japoneses não são protetores para o DMl fulminante. Em relação à parte imunológica, estudos recentes têm mostrado que entre linfócitos periféricos de pacientes com DMl fulminante foram identificadas células Thl reativas ao GAD ou a fração $\mathrm{B} 9-23$ da cadeia de insulina (33), bem como insulite foi demonstrada em autópsia de um caso fatal de DMl fulminante (34). Assim, esses achados sugerem que a etiologia do DMl fulminante possa ser heterogênea e, em uma parcela, ser auto-imune. Alguns achados sugerem que a recorrência de infecções enterovirais pode ser um dos fatores desencadeantes para o desenvolvimento do DMl fulminante, como a alta freqüência de sintomas gripais antes do seu início, a descrição de dois casos de DMl fulminante que se desenvolveram após a reativação do vírus- 6 herpes humano ou a infecção com o vírus herpes simples $(35,36)$ e a elevação de anticorpos IgA contra enterovírus em pacientes com DMl fulminante (37). Um fato que chama a atenção é que quase a totalidade descrita dos casos de DMl fulminante, até o momento, é em população japonesa ou no máximo em países asiáticos. De modo que a publicação de casos de DMl fulminante em outras populações deve colaborar para a compreensão desse subtipo de DMl que se apresenta com alterações metabólicas agudas e graves.

Tabela 1. Critérios diagnósticos para o diabetes tipo 1 fulminante.

1. Ocorrência de cetose ou CAD rapidamente após (em torno de 7 dias) do início dos sinais e sintomas de hiperglicemia

2. Glicemia $>288 \mathrm{mg} / \mathrm{dL}(16,0 \mathrm{mmol} / \mathrm{L})$ e $\mathrm{HbAlc}<8,5 \%$ ao diagnóstico

3. Peptídeo-C de jejum $<0,1 \mathrm{nmol} / \mathrm{L}$ e peptídeo-C $<0,17 \mathrm{nmol} / \mathrm{L}$ após estímulo

Committee on the Study of Fulminant Type 1 Diabetes, Japan Diabetes Society (32). 


\section{DIABETES TIPO 1 ADULTO ATÍPICO OU COM TENDÊNCIA À CETOSE (DATC)}

Há aproximadamente 15 anos, uma forma de DATC, inicialmente observada em negros americanos jovens (38) foi sugerida como uma nova entidade clínica. Esta condição se caracteriza pelo diagnóstico inicial em CAD, um período inicial de necessidade de insulina, ausência dos marcadores imunológicos do DMlA, anos de independência de insulina exógena, como observado no DM2, e episódios de CAD associados a situações de estresse (38). Este tipo de diabetes é mais freqüentemente observado em adultos obesos afro-americanos $(39,40)$, africanos do Subsaara (41), asiáticos $(42,43)$, hispânicos (44) e recentemente foi relatado no Brasil (45).

A ausência de mecanismos patofisiológicos bem definidos para essa condição tem levado a Associação Americana de Diabetes (ADA) (46) e a Organização Mundial de Saúde (OMS) (47) a colocá-la no grupo do DMl idiopático ou DMlB. Entretanto, a maioria dos investigadores que estudam esta forma de diabetes permanece atribuindo-lhe nomes diferentes, como diabetes tipo 1 de início adulto atípico, diabetes flatbush, diabetes com fase de dependência à insulina, diabetes idiopático e mais recentemente DATC $(42,43,48)$. Isso reflete certa insatisfação com a classificação atual.

Esta insatisfação pode ser reforçada por um estudo (44) que avaliou um grupo de adultos caucasianos de origem mediterrânea com diabetes tipo 1 atípico (ausência de auto-anticorpos e insulinopenia flutuante) $\mathrm{em}$ relação a características clínicas, função da célula-beta, tipagem do sistema HLA e presença de mutações nos genes do MODY e os comparou ao DM1A e ao DM2 clássicos. Neste estudo, os autores mostraram que esses pacientes diferiam dos DMIA, no que diz respeito à ausência de auto-imunidade contra as células-beta, à persistência de células-beta funcionantes, aos episódios de necessidade de insulina exógena e de tendência à cetose, bem como às características clínicas do DM2. Apenas um subgrupo destes poderia ser estritamente (ausência anticorpos e de haplotipos do sistema HLA relacionados ao DMlA) classificado DMlB.

O que se observa nesses pacientes é que, após a CAD inicial, a normalização da glicemia é seguida por longos períodos de euglicemia sem necessidade do tratamento insulínico. Entretanto, episódios de recidiva da CAD e de dependência à insulina, após curtos períodos de hiperglicemia, podem ser observados. Esta evolução nesses pacientes parece estar relacionada à propensão única a glicotoxicidade e a um padrão específico de comprometimento intermitente na secreção e na ação da insulina (41). A base genética para esta predisposição à glicotoxicidade não é bem conhecida.

No que diz respeito a predisposição à cetose nesses pacientes, existe um estudo que sugeriu que ela poderia estar relacionada a mutações no gene da enzima glicose6-fosfato dehidrogenase (G6PD). A deficiência na ação desta enzima, que catalisa o primeiro passo na via da pentose fosfato, levaria à diminuição na produção da NADPH (nicotinamida adenina dinucleotídeo) reduzida, que é o principal redutor celular. Embora existam outras vias metabólicas que produzam NADPH a G6PD, ela é a fonte predominante para a defesa celular contra o estresse oxidativo. A hipótese dos autores é de que alterações genéticas em um ou em múltiplos genes envolvidos, tanto na regulação da secreção de insulina como na expressão da G6PD pelas células-beta, poderiam predispor os indivíduos à cetose. Estas mutações poderiam levar à deficiência de insulina e à diminuição da defesa antioxidante mediada pela G6PD, contribuindo para um fenótipo específico de disfunção intermitente das célulasbeta. Esta hipótese ainda era mais atrativa pelo fato de a deficiência de G6PD ser uma desordem genética ligada ao $\mathrm{X}$ e afetar predominantemente o sexo masculino e ter alta prevalência em africanos (49). Entretanto, nesses pacientes com tendência à cetose, este estudo encontrou aumento na prevalência de deficiência de G6PD, mas sem mutações no gene dessa enzima. A falta do encontro de mutações no gene da G6PD nessa população com DATC sugere que a deficiência dessa enzima isoladamente não predispõe a esse tipo de diabetes. Por outro lado, esse estudo mostrou que nesses pacientes, mas não no DM2, o grau de deficiência na atividade da G6PD estava correlacionado com o grau de deficiência na secreção de insulina. Isso pode sugerir que a atividade da G6PD esteja relacionada à resposta da célula-beta aos estímulos, o que está de acordo com um estudo nacional (50) que mostrou bloqueio da resposta aguda da insulina à glicose em indivíduos não-diabéticos portadores de deficiência de G6PD.

A deficiência de G6PD poderia exacerbar a lesão oxidativa nas células-beta predispondo-as à disfunção. Os genes que controlam tanto a secreção de insulina como as defesas antioxidantes, mediadas pela G6PD, poderiam contribuir para o desenvolvimento do DATC em africanos.

No entanto, tem-se constatado que o denominado DATC seja um grupo heterogêneo. Um esquema para 
a classificação é importante com o objetivo de orientar tanto o tratamento, a curto e médio prazos, como também o prognóstico e os estudos para determinar a epidemiologia, a etiologia e a patogênese. Por último, esses dados seriam utilizados para elaborar intervenções efetivas na sua prevenção.

Os esquemas atuais nesse sentido têm como base os critérios imunológicos, a função da célula-beta, a necessidade de insulina e o IMC. Um estudo recente (51) foi realizado para comparar a acurácia e o valor preditivo de quatro desses critérios para determinar o fenótipo clínico e a dependência da insulina, em longo prazo, em um grupo multirracial de pacientes adultos que se apresentaram em CAD a um hospital geral. Este estudo avaliou 294 pacientes consecutivos em CAD (138 ao diagnóstico) e seguidos durante o período de um a cinco anos.

O critério que apresentou a melhor sensibilidade $(99,4 \%)$, especificidade $(95,9 \%)$, valor preditivo positivo $(97,1 \%)$ e valor preditivo negativo $(99,7 \%)$ para determinação do fenótipo e dependência da insulina em longo prazo foi o que avaliou a presença de auto-anticorpos contra as células-beta (GAD65, GAD67 e IA2) e a função da célula-beta (medida pela secreção de peptídeo-C após o estímulo com glucagon) dentro de duas semanas após a CAD inicial. A função da célula-beta foi definida preservada se o peptídeo-C de jejum era superior a $1 \mathrm{ng} / \mathrm{ml}$ ou $1,5 \mathrm{ng} / \mathrm{ml}$, após estímulo com glucagon. Com base nesse critério, os pacientes foram classificados em quatro grupos: $A^{+} \beta^{-}, A^{-} \beta^{-}, A^{+} \beta^{+}, A^{-} \beta^{+}$se apresentavam auto-anticorpos contra ilhotas positivos $\left(\mathrm{A}^{+}\right)$ou negativos $\left(\mathrm{A}^{-}\right)$e função das células-beta positiva $\left(\beta^{+}\right)$ou negativa $\left(\beta^{-}\right)$.

Ao analisar estes quatro grupos de DATC, de acordo com as classificações mais utilizadas para o diabetes atualmente, ou seja, a da ADA (46) e a da OMS (47), verifica-se que:

\section{DATC A+ $\boldsymbol{\beta}^{-}$}

O grupo DATC $A^{+} \beta^{-}$seria o tipo DMIA, ou seja, que apresenta falência das células-beta permanente e completa com a presença dos marcadores sorológicos de auto-imunidade contra essas células. Estes indivíduos necessitam de terapêutica com insulina exógena para sobrevivência.

\section{DATC $\mathbf{A}^{-} \boldsymbol{\beta}^{-}$}

O grupo DATC $\mathrm{A}^{-} \beta^{-}$seria o tipo DMlB, que também apresenta falência das células-beta permanente e com- pleta, mas não possui os marcadores de auto-imunidade contra essas células. Este grupo, à semelhança do grupo anterior, também necessita de terapêutica com insulina exógena pelo resto da vida.

\section{DATC $\mathbf{A}^{+} \boldsymbol{\beta}^{+}$}

O grupo DATC $A^{+} \beta^{+}$é constituído por indivíduos que possuem uma parcela da função das células-beta preservadas no momento do diagnóstico, mas que apresentam os marcadores sorológicos de auto-imunidade contra estas células. Este grupo poderia ser dividido em dois subgrupos: um com uma forma de disfunção da célula-beta reversível e caracterizado por período prolongado de preservação dessas células e capacidade de descontinuar a terapêutica com insulina exógena e outro com falência progressiva das células-beta e necessitando da terapêutica permanente com insulina exógena. O primeiro subgrupo é uma forma menos agressiva do grupo $\mathrm{A}^{+} \beta^{+}$e apresenta processo auto-imune contra as células-beta lento com valores baixos de anticorpos anti-GAD. A etiologia da CAD neste grupo pode ser secundária à exposição aguda à hiperglicemia, levando à dissensibilização das ilhotas diferente da glicotoxicidade que acompanha a grande destruição das célulasbeta no DMlA (52). Enquanto a primeira condição pode ser reversível com o estabelecimento da euglicemia, a última condição é irreversível (53). O segundo subgrupo corresponde ao que se denomina LADA e que está sendo discutido em outro artigo dessa edição. É interessante comentar que quando se compara o perfil de auto-anticorpos e a gravidade da CAD desse subgrupode pacientes $\left(\mathrm{A}^{+} \beta^{+}\right)$com as do grupo $\mathrm{A}^{+} \beta^{-}(\mathrm{DMl} \mathrm{A})$, verifica-se que este último apresenta maior freqüência de anticorpos anti-IA2 e gravidade maior da CAD do que a desse subgrupo de $\left(\mathrm{A}^{+} \beta^{+}\right)$, o que reforça o conceito de que a positividade para os anti-IA2 está associada com a agressividade maior de destruição das célulasbeta no diabetes auto-imune (54).

\section{DATC A- $\boldsymbol{\beta}^{+}$}

Por último, o grupo DATC $\mathrm{A}^{-} \beta^{+}$é constituído de pacientes que apresentam função das células-beta preservada e ausência dos marcadores de auto-imunidade com estas células. A maioria (especialmente os de início recente) pode descontinuar a terapêutica com insulina exógena. Este grupo é composto muito provavelmente de portadores de DM2 com vários graus de resistência à insulina e de capacidade de secreção da célula-beta. Entretanto, no momento do aparecimento da CAD 
eles apresentam um peptídeo-C relativamente menor e hormônios contra-reguladores mais elevados quando comparados aos pacientes com hiperglicemia sem cetose (55). Deve fazer parte desse grupo o subtipo de DM2, denominado por alguns de atípico, que se desenvolve em pacientes com alta sensibilidade à glico ou lipotoxicidade ou com disregulação na secreção de glucagon.

Em resumo, o grupo de diabetes denominado DATC é um grupo heterogêneo, visto que o de maior discussão é o denominado atípico. A identificação de novos mecanismos moleculares e patofisiológicos é necessária para melhor compreensão dos defeitos parcialmente reversivos ou intermitentes desse tipo de diabetes. O que poderá levá-lo a ser reconhecido como "tipo 3" ou como "outros tipos específicos" (56).

\section{DIABETES DUPLO}

Como já foi comentado na introdução, tem surgido um número crescente de crianças e adolescentes com uma mistura dos dois tipos principais de diabetes, isto é, indivíduos obesos e/ou com sinais de resistência à insulina associada à presença de marcadores de auto-imunidade contra às células-beta. Para caracterizar essa nova forma de diabetes foi introduzido, há mais de uma década, o termo DD por autores alemães (57) e reforçado recentemente por pesquisadoras americanas (58). O DD também é chamado "diabetes híbrido", "diabetes tipo 1,5" ou "diabetes auto-imune latente do jovem" (LADY).

A presença de anticorpos antidescarboxilase do ácido glutâmico (GADA), antitirosina fosfatase (IA2) e antiinsulina (IAA) em jovens com DM2 define o DD. Estudos em crianças e adolescentes americanas (59) e alemãs (60) diagnosticadas como DM2 têm demonstrado que $35 \%$ destes apresentam pelo menos um dos auto-anticorpos contra a célula-beta. O estudo europeu também mostrou que as crianças com DM2 e ICA+ não diferiam das com DM2 sem ICA, em relação a idade, sexo, peso corpóreo, perfil lipídico, pressão arterial, valor do peptídeo-C, glicemia e $\mathrm{HbAl}$ c ao diagnóstico. De modo que não existe dúvida que existe grande sobreposição entre os fenótipos do DD e do DM2 no jovem. Diferente do que ocorre na população adulta em relação ao LADA e ao DM2. O LADA pode corresponder a aproximadamente $10 \%$ dos casos de DM2, mas a resistência à insulina e a obesidade não estão entre as principais características dos indivíduos com LADA (61) como ocorre no DD.
A comparação do perfil de anticorpos contra as células-beta no DMlA como do DD tem mostrado que neste último o número de tipos de auto-anticorpos é menor (62), o que pode estar relacionado à evolução mais lenta do processo de destruição auto-imune pancreática. Em relação à imunidade mediada pelas células $\mathrm{T}$, que possui o papel principal no processo destrutivo das células-beta (63), tem sido relatado que uma porcentagem de crianças com DD e DM2 apresentam células $\mathrm{T}$ reativas a proteínas pancreáticas $(37 \%$ e $32 \%$, respectivamente, quando comparadas com $89 \%$ dos indivíduos com DMlA) (62). Entretanto, não é esclarecida se a intensidade da resposta das células $\mathrm{T}$ aos antígenos das células-beta nos indivíduos com DD segue o mesmo padrão observado no DMlA, especialmente no que se refere à velocidade de progressão para destruição total das células-beta.

O estudo do papel do componente genético para o desenvolvimento do DD tem sido discutido e é de muito interesse. Especula-se que no DD a freqüência dos maiores componentes genéticos de susceptibilidade (genes do sistema HLA) para o DMlA estaria reduzida, enquanto poderia existir associação do DD com genes de risco para o DM2.

Os genes potencialmente mais interessantes em relação ao DD são os que, em vista da sua função, poderiam influenciar nos processos patogênicos que operam tanto no DM1A como no DM2. Entre tais genes temos o do substrato 1 do receptor de insulina (IRS- 1 ) que parece ter um papel importante na resistência à insulina que caracteriza o DM2, mas também ligado à apoptose associada ao DMlA (64). A variante mais comum do IRS-1, Arg972, está associada com o DMlA (odds ratio 2,5). Os portadores dessa substituição (Arg972) do IRS-l apresentam valores de insulina e peptídeo-C de jejum menores do que os não portadores desta variante IRS-1, sugerindo que este defeito possa ter também um papel na capacidade secretora das células-beta $(64,65)$.

Outro gene potencialmente interessante no DD poderia ser o fator de transcrição nuclear, o peroxisome proliferator-activated receptor- $\gamma$ (PPAR $\gamma$ ). Este possui um papel importante na diferenciação do adipócito e na sensibilidade à insulina. Variantes no gene PPAR $\gamma$ estão associadas com IMC menor, concentrações plasmáticas de insulina mais baixas e sensibilidade à insulina melhor em várias populações (66).

$\mathrm{O}$ gene da região do fator de transcrição-7 semelhante ao 2 (TCF7L2) no cromossomo $10 \mathrm{q} 25.2$ tem 
demonstrado contribuir substancialmente para o risco de DM2 (67). Em estudo recente (68), dois SNP (single nucleotide polymorphism), rs12255372 e rs7903146 do TCF7L2, foram analisados em 6.199 DMl com idade ao diagnóstico maior que 17 anos do Reino Unido e 7.596 controles da mesma região, e não foram encontradas evidências de associação entre esses polimorfismos do TCF7L2 e o DM1. De modo que, nesse estudo, os autores comentam que uma variante que aumenta o risco para o DM2 e pode alterar a secreção de insulina não altera a susceptibilidade para a destruição auto-imune mediada das células-beta no DMl nessa população estudada.

A região do gene associado à massa gorda e à obesidade (FTO) no cromossomo $16 \mathrm{q} 12$ foi recentemente demonstrada por contribuir para o risco de obesidade (69). O SNP rs 9939609 ( $\mathrm{T}>\mathrm{A})$ deste gene está associado com aumento no IMC de aproximadamente 0,2 $\mathrm{kg} / \mathrm{m}^{2}$ por alelo em crianças com 7 anos de idade $\left(\mathrm{p}=3 \times 10^{-5}\right)$ e de $0,4 \mathrm{~kg} / \mathrm{m}^{2}$ em crianças com 11 anos. $\mathrm{O}$ efeito da variante do gene FTO na obesidade, em particular na obesidade infantil, tornou-o um bom candidato para testar se a predisposição genética para ganho de peso pode alterar a susceptibilidade para o DMIA. Nesse sentido, foi analisado um grupo de $14.803 \mathrm{DMl}$ britânicos e controles em relação ao SNP res993609 do gene FTO e não se encontrou associação entre o FTO e o DMl (70). Este estudo encontrou que o polimorfismo rs9939609, que aumenta o risco para obesidade infantil e DM2, não altera a susceptibilidade para o DMl e nem para a idade na qual o DMl se iniciou em qualquer extensão dessa população britânica.

Estes dados reforçam a hipótese de que fatores ambientais que influenciam no desenvolvimento, tanto do DM1A como do DM2, podem também ter um papel importante no DD. Entretanto, enquanto está evidente a influência da atual epidemia mundial da obesidade no desenvolvimento do DM2, o mesmo não está claro, como esta ou outros fatores associados com a obesidade afetam ou modulam o processo que leva à destruição das células-beta.

Várias hipóteses têm sido propostas para estabelecer uma ligação entre o DM1A e o DM2. As principais hipóteses sugerem que, após o seu desencadeamento, a auto-imunidade pode ser acelerada por fatores que aumentam a demanda para a produção de insulina, como crescimento acelerado, infecções, estresse psicológico e alterações climáticas (71). Recentemente, Wilkin acres- centou a esses fatores a obesidade e a resistência à insulina na chamada hipótese aceleradora (72). Esta hipótese identifica três fatores aceleradores que determinam a velocidade na qual ocorre a diminuição da massa de células-beta pancreáticas durante a vida, quais sejam: 1 . Fatores constitucionais (genes e gestação); 2. Resistência à insulina (lipo e glicotoxicidades); e 3. Genes relacionados à resposta imunológica (sistema HLA) (72). De acordo com essa hipótese, as células-beta estressadas pela sobrecarga imposta pela resistência à insulina tornam-se mais expostas ao sistema imunológico. Esta superposição de dois fatores lesivos à célula-beta explicaria uma evolução mais rápida para o diabetes.

Esta teoria, entretanto, ainda é discutida. Um dos pontos a ser esclarecido é em que fase da história natural do DMIA a obesidade aceleraria a manifestação da doença (em uma fase precoce ou tardia), quando já houvesse uma destruiç̃ão substancial das células-beta (73), pois não existem evidências de que a insensibilidade à insulina acelere a apoptose das células-beta em indivíduos normoglicêmicos, em risco para o DM2, embora possa ter importantes efeitos secundários quando a função dessas células começa a diminuir. Estudos em parentes de DMIA têm mostrado que a resistência à insulina, avaliada pelo HOMA-R, está significantemente relacionada à progressão para o DM apenas naqueles indivíduos com perda da primeira fase de secreção de insulina (PFSI) (74). Esta controvérsia é particularmente relevante no DD, porque estes indivíduos são persistentemente obesos e resistentes à insulina, enquanto a presença dos marcadores imunológicos do DMlA representa um fator adicional coexistente, isto é, a presença das características do DMIA e do DM2 no DD sugere mais uma sobreposição parcial (dupla) do que total (única) entre os dois tipos principais de diabetes (75).

É de conhecimento geral que as adipocitoquinas e outros marcadores inflamatórios ligados à obesidade $\mathrm{e}$ à resistência à insulina possuem efeitos imunomodulatórios (76). Estes efeitos podem desencadear uma resposta imunológica pela mudança do equilíbrio na resposta Th1/Th2 linfocitária ou pelo comprometimento da função dos linfócitos $\mathrm{T}$ reguladores, isto é, das células CD4+ CD25+. Um dos principais papéis nesse sentido pode ser atribuído à leptina, que regula a ingestão alimentar e o IMC. Os valores plasmáticos de leptina possuem relação direta com a gordura corpórea total. A leptina controla a resposta da imunidade celular 
e tem sido relacionada a várias doenças auto-imunes (77). Estudos em modelos experimentais de DMlA ( $\mathrm{NOD}^{-}$camundongo não-obeso diabético) têm demonstrado que a administração de leptina a esses animais promove um infiltrado inflamatório precoce das ilhotas pancreáticas, aumenta a produção de interferon- $\gamma$ (IFN- $\gamma$ ) pelas células T e antecipa o aparecimento do DM (78). A obesidade que é uma situação de hiperleptinemia é caracterizada pela infiltração macrofágica do tecido adiposo (79).

Outro mecanismo que pode estar envolvido, nesta relação entre obesidade e interleucinas e auto-imunidade, é o aumento da expressão de auto-antígenos das células-beta (principalmente GAD) associada com a IL- 1, IFN- $\gamma$ e TNF- $\alpha$ liberado pelas ilhotas, o que favorece a vulnerabilidade dessas células à destruição autoimune (80). Nesse sentido, um estudo recente mostrou que títulos elevados de GADA estavam relacionados positivamente com o aumento no IMC (81).

Em resumo, existem evidências crescentes de que a leptina e outras citoquinas ligadas à obesidade possuem papel importante no controle da auto-imunidade, sugerindo que sinais hormonais inflamatórios associados à resistência à insulina podem amplificar a resposta imu- ne no DD. Na Figura 1, de modo esquemático, a interrelação entre a dieta hipercalórica, a obesidade e a auto-imunidade no desenvolvimento do DD [adaptação de Donath \& Halban (82)].

A suspeita do DD surge quando a hiperglicemia se inicia em crianças ou adolescentes obesos. Inicialmente, o que chama a atenção nesses jovens são as características clínicas do DM2, como hipertensão arterial, dislipidemia e IMC elevado, o que já lhes confere aumento no risco cardiovascular, quando comparados com crianças portadoras do DMlA clássico. No sexo feminino pode-se encontrar também a presença da síndrome dos ovários policísticos. $\mathrm{O}$ antecedente familiar para DMIA e DM2 podem estar presentes, bem como um quadro menos exuberante de emagrecimento, poliúria e polidpsia e de CAD. A presença dos anticorpos antiilhotas, embora em número e títulos menores do que do DMIA e provavelmente dos HLA de risco para o DMlA clássico. Os jovens com DD são caracterizados por um fenótipo obeso e a coexistência de auto-imunidade contra as células-beta. Na Figura 2, o exemplo de um jovem classificado como DD no Centro de Diabetes da Universidade Federal de São Paulo/Escola Paulista de Medicina (Unifesp).

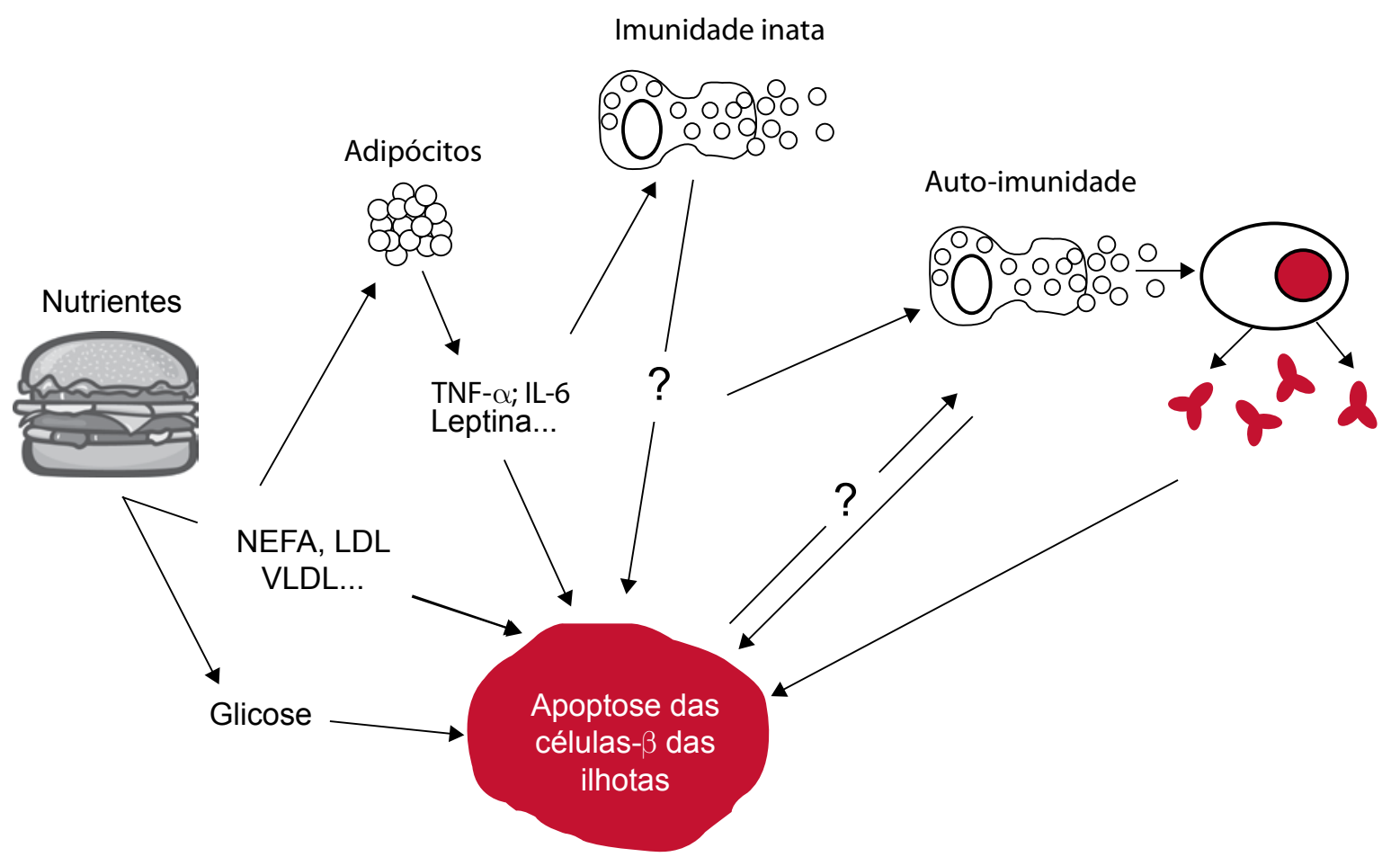

Figura 1. Inter-relação entre dieta hipercalórica, obesidade e auto-imunidade no desenvolvimento do DD. 
Dib

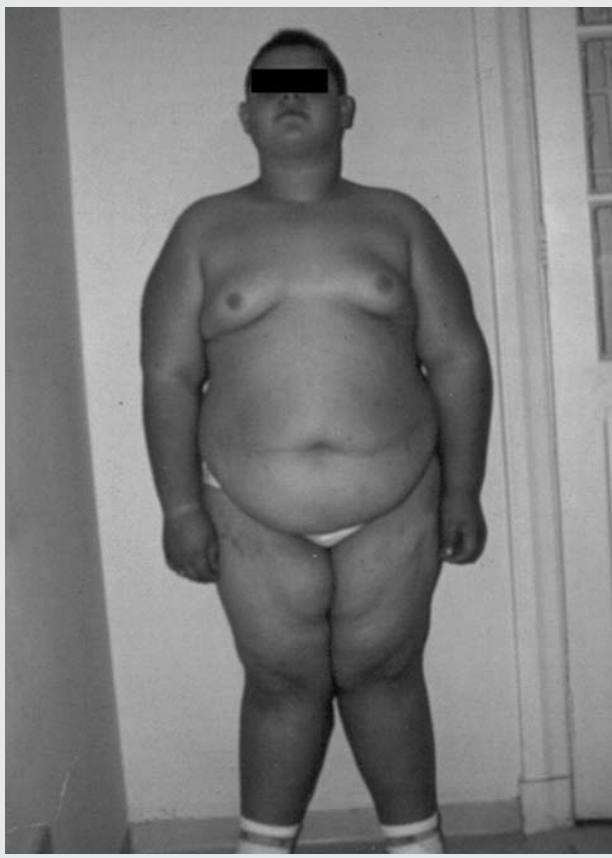

- Id. TGS;

- Idade ao diagnóstico: 14 anos:

- Antecedente familiar: avó e mãe com DM2;

- Diagnóstico em CAD:

- Glicemia: 535 mg/dL;

- Cetonuria: +;

- $\mathrm{HCO}^{3}: 10 \mathrm{mE} / \mathrm{L}$;

- IMC: $43 \mathrm{~kg} / \mathrm{m}^{2}$

- Acanthosis nigricans: cervical e axilar;

- Hipertensão arterial;

- Peptídeo-C de jejum: 1,7 ng/ml (vn: 0,6 a 3,6 ng/ml);

- GADA: $20 \mathrm{Ku} / \mathrm{ml}(\mathrm{vn}:<1,7)$;

- Anti-IA2: 14,2 Ku/ml (vn: <0,5);

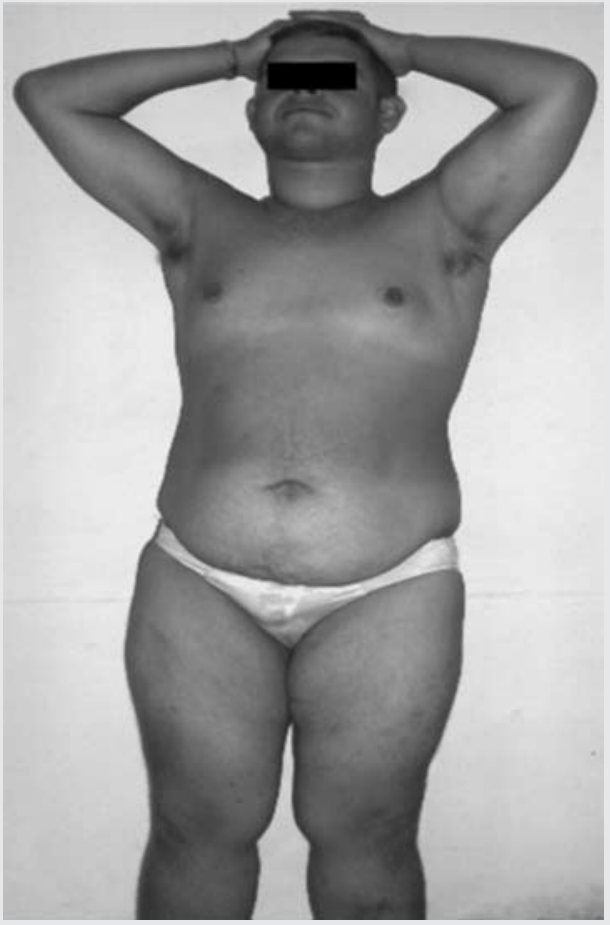

\section{- Evolução (7,5 anos de seguimento).}

- Terapêutica:

- Dieta para DM2 no jovem;

- Insulina;

- Metformina;

- Enalapril;

- IMC: $31,9 \mathrm{~kg} / \mathrm{m}^{2}$;

- CA: $103 \mathrm{~cm}$;

- Pep-C J: 3,1 ng/ml e GADA: 17,5 U/ml.

Figura 2. Jovem com DD.

Considerando que o DD apresenta uma função residual de células-beta significativa no momento do diagnóstico e que a queda na sua função pode ser mais lenta do que no DMlA clássico, as intervenções capazes de interferir com os mecanismos envolvidos no processo da doença são potencialmente interessantes, bem 
como as medidas relacionadas ao tratamento clássico do DM2, como dieta, exercício para redução do peso e intervenções farmacológicas com drogas e insulina.

A redução do peso e/ou exercício reduzem acentuadamente os níveis de marcadores inflamatórios circulantes, como a proteína-C reativa (PCR) e a IL-6, como a insulinoterapia tem impacto nos valores de PCR, IL-1, IL-6, TNF- $\alpha$, e PAI- 1 e a metformina na PCR. Outras drogas, como as glitazonas e as incretinas, também possuem potencial nesse sentido. Estas ações poderão repercutir também na infiltração inflamatória do tecido adiposo (83). Do mesmo modo se pode sugerir que estas alterações tenham influência favorável no processo da doença reduzindo a destruição auto-imune progressiva das células-beta (limitando o aumento dos títulos dos auto-anticorpos e na porcentagem de células $\mathrm{T}$ que se desviam para o padrão de resposta Thl) nos indivíduos que emagrecem, resultando uma preservação maior da massa de células-beta pancreáticas.

Estes jovens com DD devem ser abordados, em paralelo, em relação a outras comorbidades que possam estar associadas, como hipertensão arterial e dislipidemia, pois existem evidências de que a prevenção primária das doenças cardiovasculares deve ser iniciada na infância e na juventude (84), e os pacientes com DD apresentam grande risco de desenvolvê-las precocemente.

Nesse sentido, encontra-se também na literatura a denominação de "DD" (85) para aqueles pacientes com DMlA clássico no início, mas que, com a evolução da doença adquirem características da síndrome metabólica (SM) e aumento no risco de desenvolvimento das doenças cardiovasculares.

No nosso meio, analisou-se um grupo de 521 pacientes com DMl, mediana de idade igual a 18 anos (3 a 55 anos) e tempo médio de diagnóstico do diabetes de 6 anos (0,17 a 40 anos). Nesse estudo, verificou-se que a prevalência de SM variou de $12 \%$ a $19 \%$, dependendo do critério utilizado (86). Os fatores que se relacionaram diretamente com a sua presença foram a idade cronológica, o sexo feminino, o tempo de diagnóstico clínico da doença, o valor da HbAlc e a presença de microalbuminúria (87).

O estudo citado anteriormente (85), que sugeriu também a denominação de "DD", foi realizado com os pacientes do conhecido estudo do Diabetes Control and Complications Trial (DCCT). Este estudo mostrou que os pacientes com os valores mais elevados de resistência à insulina, avaliados pelo estimated glucose disposal rate (eGDR), na sua avaliação inicial, foram os que apresentaram o risco maior de desenvolvimento das doenças micro e macrovasculares, independentemente do tratamento instituído (intensivo ou convencional).

Estes estudos reforçam o conceito atual de que a abordagem do DMl deve passar de glicocêntrica para multifatorial e precoce em relação ao sedentarismo, excesso de peso, dislipidemia, hipertensão arterial e microalbuminúria.

Do ponto de vista clínico e de saúde pública, a identificação de pacientes com DMl e características da SM é útil para selecionar candidatos a tratamentos específicos (dislipidemia, hipertensão arterial, obesidade visceral) que poderiam não estar sendo aplicados naquele momento, mas que são de importância comprovada para o prognóstico e na qualidade de vida dos indivíduos com DM.

\section{CONSIDERAÇÕES FINAIS}

Os avanços na área da genética, das biologias molecular e celular, da imunologia e da bioquímica têm mostrado que o DMl é um grupo de etiologia heterogênea.

Dentro dessa heterogeneidade têm-se desde formas mais raras de DMl auto-imune até quadros mistos e em todas as faixas etárias.

$\mathrm{Na}$ caracterização dos processos não-auto-imunes que provocam a destruição da célula-beta, com exceção dos agentes infecciosos, metabólicos, álcool e metais pesados que não foram discutidos neste artigo, é importante avaliar o grupo racial, alterações em mecanismos importantes para a secreção de insulina e sobrevida das células-beta, o comprometimento da parte exócrina pancreática, a ausência dos marcadores imunológicos da doença, o modo de início do DM e o fenótipo do indivíduo.

As mudanças no perfil antropométrico (aumento da prevalência de obesidade) e no estilo de vida (aumento do sedentarismo) e o diagnóstico precoce da presença da auto-imunidade contra as células-beta têm possibilitado o aparecimento de uma sobreposição entre os dois tipos mais freqüentes do DM (DMIA e DM2) no mesmo indivíduo, caracterizando o DD. A influência das adipoquinas, relacionadas à obesidade, 
na velocidade da evolução do processo auto-imune tem sido discutida.

O conhecimento desta heterogeneidade pode fazer diferença nas decisões de como, quando e quem deveria participar dos estudos de prevenção de modo mais freqüente do DMl, isto é, a auto-imune, e na abordagem das formas atípicas ou híbridas da doença.

Entretanto, até que meios mais práticos e abrangentes para a classificação dos tipos de diabetes estejam disponíveis, é importante para o clínico tratar individualmente o seu paciente com base no seu quadro clínico, fenótipo e características bioquímicas.

\section{REFERÊNCIAS}

1. Eisenbarth GS. Update in type 1 diabetes. J Clin Endocrinol Metab. 2007;92(7):2403-7.

2. Barker JM. Clinical review: type 1 diabetes-associated autoimmunity: natural history, genetic associations and screening. $\mathrm{J}$ Clin Endocrinol Metab. 2006;91(4):1210-7.

3. Fourlanos S, Perry C, Stein MS, Stankovich J, Harrison LC, Colman PG. A clinical screening tool identifies autoimmune diabetes in adults. Diabetes Care. 2006;29(5):970-5.

4. Su MA, Anderson MS. Aire: an update. Curr Opin Immunol. 2004; $16: 746-52$.

5. Wildin RS, Freitas A. IPEX and FOXP3: clinical and research perspectives. J Autoimmun. 2005;25:(Suppl):56-62.

6. Hagopian WA, Sanjeevi CB, Kockum I, Landin-Olsson M, Karlsen $A E$, Sundkist G, et al. Glutamate decarboxylase-, insulin-, and islet cell-antibodies and HLA typing to detect diabetes in a general population-based study of Swedish children. J Clin Invest. 1995;95:1505-11.

7. Maldonado M, Hampe CS, Gaur L, D'Amico S, lyer D, Hammerle LP, et al. Ketosis-prone diabetes: dissection of a heterogeneous syndrome using an immunogenetic and beta-cell functional classification, prospective analysis and clinical outcomes. J Clin Endocrinol Metab. 2003; 88(11):5090-8.

8. Imagawa A, Hanafusa T, Uchigata Y, Kanatsuka A, Kawasaki E, Kobayashi $T$, et al. Fulminant type 1 diabetes a nationwide survey in Japan. Diabetes Care. 2003;26(8):2345-52.

8a. Molven A, Ringdal M, Nordbo AM, Rader H, Stay J, Lipkind $\mathrm{GM}$, et al. Mutations in the insulin gene can cause MODY and autoantibody-negative type 1 diabetes. Diabetes. 2008. Jan 11. (Epub ahead of print)

9. American Diabetes Association. Type 2 diabetes in children and adolescents. American Diabetes Association. Diabetes Care. 2000;23:381-9.

10. Sosenko JM, Krischer JP, Palmer JP, Mahon J, Cowie C, Greenbaum $C J$, et al. A risk score for type 1 diabetes derived from autoantibody positive participants in the diabetes prevention trial - type 1. Diabetes Care. 2007. Publish ahead of print, published online December 4.

11. Libman IM, Becker DJ. Coexistence of type 1 and type 2 diabetes mellitus: "double" diabetes? Pediatr Diabetes. 2003;4: 110-3.
12. Nagamine K, Peterson P, Scott HS, Kudoh J, Minoshima S, Heino $M$, et al. Positional cloning of the APECED gene. Nat Genet. 1997;17:393-8.

13. Björses P, Pelto-Huikko M, Kaukonen J, Aaltonen J, Peltonen L, Ulmanen I. Localization of the APECED protein in distinct nuclear structures. Hum Mol Genet. 1999;8:259-66.

14. Meager $A$, Visvalingam $K$, Peterson $P$, et al. Anti-interferon antibodies in autoimmune polyendocrine syndrome type 1. Plos Medicine. 2006;3:e289.

15. Notkins AL, Lernmark $\AA$. Autoimmune type 1 diabetes: resolved and unresolved issues. J Clin Invest. 2001;108:1247-52.

16. Gylling M, Tuomi T, Björses P, Kontiainen S, Partanen J, Christie $M R$, et al. Beta-cell autoantibodies, human leukocyte antigen II alleles, and type 1 diabetes in autoimmune polyendocrinopathycandidiasis - ectodermal dystrophy. J Clin Endocrinol Metab. 2000;85:4434-40.

17. Powell BR, Buist NR, Stenzel P. An X-linked syndrome of diarrhea, polyendocrinopathy, and fatal infection in infancy. $\mathrm{J} \mathrm{Pe}$ diatr. 1982;100:731-7.

18. Godfrey VL, Wilkinson JE, Russel LB. X-linked lymphoreticular disease in the scurfy (SF) mutant mouse. Am J Pathol. 1991;138:1379-87.

19. Chatila TA, Blaeser F, Ho N, et al. JM2, enconding a fork headrelated protein, is mutated in X-linked autoimmunity-allergic disregulation syndrome. J Clin Invest. 2000;106:R75-R81.

20. Walker MR, Kasprowicz DJ, Gersuk VH, et al. Induction of FoxP3 and acquisition of $\mathrm{T}$ regulatory activity by stimulated human CD4+CD25- T cells. J Clin Invest. 2003;112:1437-43.

21. Baud $O$, Goulet $O$, Canioni $D$, et al. Treatment of the immune dysregulation, polyendocrinopathy, enteropathy, X-linked syndrome (IPEX) by allogenic bone marrow transplantation. $\mathrm{N}$ Engl J Med. 2001;344:1758-62.

22. Bindl L, Torgerson T, Perroni $L$, et al. Successful use of the new immunesupressor sirolimus in IPEX (immune dysregulation, polyendocrinopathy, enteropathy, X-lonked syndrome). J Pediatr. 2005;147:256-9.

23. Bingley PJ, Bonifacio E, Williams AJK, Genovese S, Bottazzo GF, Gale EAM. Prediction on IDDM in the general population; strategies based on combinations of autoantibody markers. Diabetes 1997:46:1701-10.

24. Tiberti C, Buzzetti R, Anastasi E, Dotta F, Vestal M, Petrone A, et al. Autoantibody negative new onset type 1 diabetic patients lacking high risk HLA alleles in a Caucasian population: are type $1 \mathrm{~b}$ diabetes cases? Diabetes Metab Res Rev. 2000; 16:8-14.

25. Wang J, Miao D, Babu S, Yu J, Barker J, Klingensmith G, et al. Prevalence of autoantibody-negative diabetes is not rare at all ages and increases with older age and obesity. J Clin Endocrinol Metab. 2007;92(1):88-92.

26. Wenzlau JM, Juhl K, Yu L, Moua O, Sarka SA, Gottlieb P, et al. The cation efflux transporter $\mathrm{ZnT8}$ (SIc30A8) is a major autoantigen in human type 1 diabetes. PNAS. 2007; 104(43): 17040-5.

27. Imagawa A, Hanafusa T, Miyagawa J, Matsuzawa Y. A novel subtype of type 1 diabetes mellitus characterized by a rapid onset and an absence of diabetes-related antibodies. Osaka IDDM study group. N Engl J Med. 2000;342:301-7.

28. Wakisaka M, Nunoi K, Wada M, et al. Two cases of abrupt onset type 1 diabetes associated with elevation of elastase 1 and diabetic ketoacidosis. J Jpn Diab Soc. 1987;30:619-24.

29. Kawasaki E, Matsura N, Eguchi K. Type 1 diabetes in Japan. Diabetologia. 2006;49(5):828-36. 
30. Sayama K, Imagawa A, Okita K, Uno S, Moriwaki M, Kozawa J, et al. Pancreatic beta and alpha cells are both decreased in patients with fulminant type 1 diabetes: a morphometrical assessment. Diabetologia. 2005;48:1560-4.

31. Imagawa $A$, Hanafusa $T$, Uchigata $Y$, et al. Fulminant type 1 diabetes: a nationwide survey in Japan. Diabetes Care. 2003;26:2345-52.

32. Kawasaki E, Eguchi K. Is type 1 diabetes in the Japanese population the same as among Caucasians? Ann NY Acad Sci. 2004;1037:96-103.

33. Kotani $R$, Nagata $M$, Imagawa $A$, et al. T lymphocyte response against pancreatic beta cell antigens in fulminant type 1 diabetes. Diabetologia. 2004;47:1285-91.

34. Tanaka S, Kobayashi T, Momotsu T. A novel subtype of type 1 diabetes mellitus. N Engl J Med. 2000;342:1835-7.

35. Sekine N, Motokura T, Oki T, et al. Rapid loss of insulin secretion in a patient with fulminant type 1 diabetes mellitus and carbamazepine hypersensitivity syndrome. JAMA. 2001;285: 1153-4.

36. Nagaoka T, Terada M, Miyakoshi H. Insulin-dependent diabetes mellitus following acute pancreatitis caused by herpes simplex virus: a case report. J Jpn Diab Soc. 2001;44:335-40.

37. Imagawa A, Hanafusa T, Makino H, Miyagawa JI, Juto P. High titres of $\lg A$ antibodies to enterovirus in fulminant type- 1 diabetes. Diabetologia. 2005;48:290-3.

38. Winter WE, Maclaren NK, Riley WJ, et al. Maturity-onset diabetes of youth in black Americans. N Engl J Med. 1987;316:285-91.

39. Banerji MA, Chaiken RL, Huey H, Tuomi T, Norin AJ, Mackay IR, et al. GAD antibody negative NIDDM in adult black subjects with diabetic ketoacidosis and increased frequency of human leukocyte antigen DR3 and DR4: Flatbush diabetes. Diabetes. 1994;43:741-5.

40. Pinero-Pilona A, Litonjua P, Aviles-Santa L, Raskin P. Idiopathic type 1 diabetes in Dallas, Texas: a 5-year experience. Diabetes Care. 2001;24:1014-8.

41. Mauvais-Jarvis F, Sobngwi E, Porcher R, Riveline J-P, Kevorkian J-P, Vaisse C, et al. Ketosis-prone type 2 diabetes in patients of sub-saharan african origin clinical pathophysiology and natural history of beta-cell dysfunction and insulin resistance. Diabetes. 2004;53:645-53.

42. Tanaka K, Moriya T, Kanamori A, Yajima Y. Analysis and a long-term follow-up of ketosis-onset Japanese NIDDM patients. Diabetes Res Clin Pract. 1999;44:137-46.

43. Tan KC, Mackay IR, Zimmet PZ, Hawkins BR, Lam KS. Metabolic and immunologic features of Chinese patients with atypical diabetes mellitus. Diabetes Care. 2000;23:335-8.

44. Aguilera E, Casamitjana R, Ercilia G, Oriola J, Gomes R, Conget I. Adult-onset atypical (type 1) diabetes. Additional insights and differences with type $1 \mathrm{~A}$ diabetes in a European Mediterranean population. Diabetes Care. 2004;27:1108-14.

45. Rodacki M, Zajdenverg L, Lima GAB, Nunes RC, Milech A, Oliveira JEP. Relato de caso: Diabetes flatbush - cetoacidose ao tratamento não farmacológico - Diabetes ketosis prone. Arq Bras Endocrinol Metab. 2007;51(1):131-5.

46. Expert Committee on the Diagnosis and Classification of Diabetes Mellitus. Report of the Expert Committee on the Diagnosis and Classification of Diabetes Mellitus. Diabetes Care. 1997;20:1183-97.

47. Alberti KG, Zimmet PZ. Definition, diagnosis and classification of diabetes mellitus and its complications. Part 1: diagnosis and classification of diabetes mellitus provisional report of a WHO consultation. Diabet Med. 1998;15:539-53.

48. Pinero-Pilona A, Litonjua P, Aviles-Santa L, Raskin P. Idiopathic type 1 diabetes in Dallas, Texas: a 5-year experience. Diabetes Care. 2001;24:1014-8.

49. Beutler E. G6PD deficiency. Blood. 1994;84:3613-36.

50. Monte Alegre S, Saad ST, Delatre E, Saad MJ. Insulin secretion in patients deficient in glucose-6-phosphate dehydrogenase. Horm Metab Res. 1991;23:171-3.

51. Balasubramanyam A, Gaza G, Rodriguez L, Hampe CS, Gaur L, Lernmark $A$, et al. Accuracy and predictive value of classification schemes for ketosis-prone diabetes. Diabetes Care. 2006;29:2575-9.

52. Mathis D, Vence L, BenoistC. Beta-cell death during progression to diabetes. Nature. 2001;414:792-8.

53. Poitout P, Robertson PR. Glucolipotoxicity: fuel excess and beta-cell dysfunction. Endocrine Reviews. 2007; First published ahead of print November 29.

54. Borg H, Gottsater A, Fernlund P, Sundkvist G. A 12-year prospective study of the relationship between islet antibodies and beta-cell function at and after the diagnosis in patients with adult-onset diabetes. Diabetes. 2002;51:1754-62.

55. Umpierrez GE, Kelly JP, Navarrete JE, Casals MC, Kitabchi AE. Hyperglycemic crises in urban blacks. Arch Intern Med. 1997; 157:669-75.

56. Sobngwi E, Gautier JF. Adult-onset idiopathic type I or ketosisprone type II diabetes: evidence to revisit diabetes classification. Diabetologia. 2002;45:283-5.

57. Teupe B, Bergis K. Epidemiological evidence for "double diabetes". Lancet. 1991;337(9):361-2.

58. Libman IM, Becker DJ. Coexistence of type 1 and type 2 diabetes mellitus: "double" diabetes? Pediatr Diabetes. 2003;4:110-3.

59. Hathout EH, Thomas W, El-Shahawy M, Nahab F, Mace JW. Diabetic autoimmune markers in children and adolescents with type 2 diabetes. Pediatrics. 2002;107(6):1-4.

60. Reinehr T, Schober E, Wiegand S, Thon A, Holl R. On behalf of the DPV-wiss study group. Beta-cell autoantibodies in children with type 2 diabetes mellitus: subgroup or misclassification? Arch Dis Child. 2006;91:473-7.

61. Vadacca M, Valeri C, Gigante A, Guglielmi C, Pozzilli P. Action LADA group. Gender differences and the metabolic syndrome in patients with type 2 diabetes with or without autoantibodies to glutamic acid decarboxilase (GAD). Diabetes. 2006;55(Suppll.1)Abst.1204-P, A283.

62. Brooks-Worrell BM, Greenbaum CJ, Palmer JP, Pihoker C. Autoimmunity to islet proteins in children diagnosed with newonset diabetes. J Clin Endocrinol Metab. 2004(89):2222-7.

63. Bisikirska BC, Herold KC. Regulatory T cell and type 1 diabetes. Curr Diab Rep. 2005;5:104-9.

64. Sesti G, Federici M, Hribal ML, Lauro D, Sbraccia P, Lauro R. Defects of the insulin receptor substrate (IRS) system in human metabolic disorders. FASEB J. 2001;15:2099-111.

65. Federici M, et al. The common Arg972 polymorphism in insulin receptor substrate-1 causes apoptosis of human pancreatic islets. FASEB J. 2001;15:22-4.

66. Altshuler D, et al. The common PPARg Pro12Ala polymorphism is associated with decreased risk of type 2 diabetes. Nat Genet. 2000;26:76-80.

67. Grant SFA, Thorleifsson G, Reynisdottir I, Benediktsson R, Manolescu A, Sainz J, et al. Variant of transcription factor 7-like 2 
(TCF7L2) gene confers risk of type 2 diabetes. Nat Genet. 2006;38:320-3.

68. Field SF, Howson JMM, Smyth DJ, Walker NM, Dunger DB, Todd JA. Analysis of the type 2 diabetes gene, TCF7L2, in 13,795 type 1 diabetes cases and control subjects. Diabetologia. 2007;50:212-3.

69. Frayling TM, Timpson NJ, Weedon MN, Zeggini E, Freathy $\mathrm{RM}$, Lindgren CM, et al. A common variant in the FTO gene is associated with body mass index and predisposes to childhood and adult obesity. Science. 2007;316(5826):889-94.

70. Field SF, Howson JMMM, Walker NM, Dunger DB, Todd JA. Analysis of the obesity gene FTO in 14,803 type 1 diabetes cases and controls. Diabetologia. 2007;50(10):2218-20.

71. Dahlquist GG. Environmental risk factors in human type1 diabetes - an epidemiological perspective. Diabetes Metab.1995; 11:37-46.

72. Wilkin TJ. The accelerator hypothesis: weight gain as the missing link between type I and type II diabetes. Diabetologia. 2001;44:914-22.

73. Dabelea $D$, et al. Testing the accelerator hypothesis: body size, beta-cell function, and age at onset of type 1 (autoimmune) diabetes. Diabetes Care. 2006;29:290-4.

74. Tsai EB, Sherry NA, Palmer JP, Herold KC. The rise and fall of insulin secretion in type 1 diabetes mellitus. Diabetologia. 2006;49:261-70.

75. Gale EAM. To boldly go - or to go too boldly? The accelerator hypothesis revisited. Diabetologia. 2007;50:1571-5.

76. Aldhahi W, Hamdy O. Adipokines, inflammation, and the endothelium in diabetes. Curr Diab Rep. 2003;3:293-8.

77. Lord G. Role of leptin in immunology. Nutr Rev. 2002;60:S5-S38.

78. Matarese G, et al. Leptin in immunology. J Immunol. 2005;174: 3137-42.

79. Weisberg SP, et al. Obesity is associated with macrophage accumulation in adipose tissue. J Clin Invest. 2003;112: 1796-808.

80. Wachlin G, et al. IL-1b, IFN-b and TNF-a increase vulnerability of pancreatic $\beta$ cells to autoimmune destruction. J Autoimmun. 2003;20:303-12.

81. Rolandsson $O$, et al. Glutamate decarboxylase (GAD65) and tyrosine phosphatase-like protein (IA-2) autoantibodies index in a regional population is related to glucose intolerance and body mass index. Diabetologia. 1999;42:555-9.

82. Donath MY, Halban PA. Decreased beta-cell mass in diabetes: significance, mechanisms and therapeutic implications. Diabetologia. 2004;47:581-9.

83. Kolb H, Mandrup-Poulsen T. An immune origin of type 2 diabetes. Diabetologia. 2005;48:1038-50.

84. McCrindle BW, Urbina EM, Dennison BA, Jacobson MS, Steinberger, J, Rocchini AP, et al. Drug therapy of high - risk lipid abnormalities in children and adolescents. A scientific statement from the American Heart Association Atherosclerosis, Hypertension, and Obesity in Youth Committee, Council of Cardiovascular Disease in the Young, With the Council on Cardiovascular Nursing. Circulation. 2007;115:1948-67.

85. Kilpatrick ES, Rigby AS, Atkin SL. Insulin resistance, the metabolic syndrome, and complication risk in type 1 diabetes "double diabetes" in the diabetes control and complications trial. Diabetes Care. 2007;30:707-12.

86. Pires AC, Cordeiro JA, Gabbay MAL, Giuffrida FMA, Gomes MB, DibSA. Prevalence of metabolic syndrome in young type 1 diabetes: do we need a specific criterion? Diabetes. 2006;55(Suppl1):abstract 1774 - P.A410.

87. Dib SA. Síndrome metabólica no diabetes melito do tipo 1 [tese de livre docência]. São Paulo: Universidade Federal de São Paulo. Escola Paulista de Medicina, 2006.

Endereço para correspondência:

Sergio Atala Dib

Unifesp - Disciplina de Endocrinologia

Rua Botucatu, 740

04023-900 - Vila Clementino, São Paulo, SP

E-mail: sergio.dib@unifesp.br 